\title{
Cyclin D and cyclin E expression in normal and adenomatous pituitary
}

\author{
Suzanne Jordan ${ }^{1}$, Kulbir Lidhar ${ }^{1}$, Marta Korbonits, David G Lowe ${ }^{1}$ and Ashley B Grossman \\ Department of Endocrinology and ${ }^{1}$ Department of Histopathology, St Bartholomew's Hospital, London EC1A 7BE, UK \\ (Correspondence should be addressed to A B Grossman; Email: A.B.Grossman@mds.qmw.ac.uk) \\ (S Jordan and K Lidhar contributed equally to this work)
}

\begin{abstract}
Objectives: Cyclins play an important role in the regulation of cell progression through the cell cycle. Over-expression of the cyclins has been shown in many different tumour types. Pituitary adenomas are a common form of endocrine neoplasia in the human, but have been little studied in terms of the expression of the principal cyclins regulating checkpoint exit, cyclin D1 and cyclin E.

Methods: We therefore investigated the expression of cyclin D1 and cyclin E in a range of benign and metastatic pituitary tumours. We studied a total of 95 pituitaries, including normal pituitary $(n=20)$, Cushing's disease $(n=19)$, somatotroph tumours $(n=19)$, non-functioning adenomas $(n=18)$, prolactinomas $(n=7)$, aggressive tumours $(n=9)$ and pituitary carcinoma $(n=3)$. All tumours and normal tissue were immunostained for cyclin D1 and cyclin E using a standard technique, and were then subjected to blinded analysis by a single observer and the extent of staining quantified on the basis of 500 cell counts per tissue. The distribution of positive staining between different tissues was analysed by non-parametric test procedures.

Results: There was no cytoplasmic staining for cyclin D1 in any tissue. Nuclear staining was generally sparse, but was statistically more frequent in non-functioning and aggressive tumours compared with other tumour types or normal pituitary. Cyclin E was also sparsely expressed, but was specifically increased in corticotroph tumours from patients with Cushing's disease.

Conclusions: We report cyclin D1 over-expression in aggressive and non-functioning pituitary tumours, and that cyclin E espression is more frequently seen in Cushing's disease. The high level of cyclin E expression in Cushing's disease may relate to the low level of p27 protein expression previously reported in corticotroph tumours.
\end{abstract}

European Journal of Endocrinology 143 R1-R6.

\section{Introduction}

Cyclins and cyclin-dependent kinases (CDKS) are essential for cell cycle regulation in eukaryotes. Cyclins, the regulatory subunits, bind to CDKS, the catalytic subunits, to form active cyclin-CDK complexes. Active cyclin-CDK complexes drive cells through particular cell cycle phases, called 'check points', by phosphorylating the unique sets of protein substrates that are essential to achieve transition to the next phase. The levels of cyclins are regulated at the level of transcription as well as by targeted degradation via the ubiquitin pathway (1).

Cyclin D1 is a protein coded for by the PRAD1-CCND1 or bcl-1 gene. During the G1 phase of the cell cycle, cyclin D1, together with its CDK partners (CDK4 and CDK6), is responsible for the transition to $S$ phase by phosphorylating the product of the retinoblastoma gene (pRB), which in turn releases transcription factors important in the initiation of DNA replication. Several lines of evidence link aberration of the cell cycle to tumorigenesis (reviewed in 2-4). Most particularly, dysregulation of cyclin D1 synthesis allows cell cycle progression in the absence of growth factors and may contribute to the initiation of oncogenesis. For example, over-expression of cyclin D1 has been reported in various human malignant tumours, including 30\% of oesophageal cancers (5). In breast cancers, the cyclin D1 gene is amplified in $20 \%$ of cases, and also in $10 \%$ of hepatocellular carcinomas (6). Cyclin D1 has also been implicated as an oncogene in parathyroid adenomas, with the cyclin D1 gene rearranged in relation to the parathyroid hormone gene locus (7). In addition, cyclin D1 is diagnostically over-expressed in mantle cell lymphomas (8). Many cancers have been reported to harbour amplifications of the chromosome band 11q13, where cyclin D1 is located (5). 
Human cyclin E was discovered by its ability to rescue $S$. cerevisiae cells lacking G1 cyclin function. Cyclin E forms a complex exclusively with CDK2 and activates this serine-threonine kinase at the restriction point shortly prior to entry into S phase (9). As for cyclin D1, there is evidence supporting a role for cyclin $\mathrm{E}$ as an oncogene, suggesting that deregulation of this protein is one of the primary events which alters the regulation of checkpoint controls in normal cells, leading to a tumour phenotype. Over-expression has been associated with poorly differentiated and invasive hepatocellular carcinoma when associated with p53 mutations. Overexpression of the cyclin $\mathrm{E}$ gene in breast cancer has also been demonstrated, with alterations of cyclin $\mathrm{E}$ becoming more severe with increasing breast tumour stage and grade. These findings support the use of cyclin $\mathrm{E}$ as a powerful prognostic marker for breast cancer (10).

In spite of the extensive data on these cyclins in a variety of tumour phenotypes, there are currently only limited data on cyclin D and E expression in pituitary tumours. Recently, one group has suggested that cyclin D may be over-expressed in non-functioning pituitary tumours compared with normal pituitary (11). Various groups have recently reported that the tumour suppressor p27, which acts as a CDK inhibitor, is significantly decreased in pituitary adenomas at the protein level as compared with normal pituitary $(12-14)$, in spite of normal or even increased levels of p27 mRNA expression (15); its protein expression is diminished still further in corticotroph adenomas and malignant pituitary tumours (14). The aim of the current study was therefore to investigate whether over-expression of cyclins D1 and E occurs in pituitary tumours compared with the normal pituitary gland, whether different pituitary tumour types and tumour behaviour express differences in cyclin D or cyclin E expression, and to relate any possible differences to $\mathrm{p} 27$ expression.

\section{Materials and methods}

\section{Tumour specimens}

We studied 95 pituitary tissue samples removed at transsphenoidal surgery. These were classified histologically into 20 normal pituitaries, 63 pituitary adenomas, 9 aggressive tumours and 3 metastatic pituitary tumours. The normal pituitaries were resection specimens removed at transsphenoidal surgery for presumptive tumours that proved on standard haematoxylin and eosin, immunohistochemical and reticulin staining to consist of normal pituitary tissue and architecture; these have been previously reported in terms of p27-immunoreactivity, and were not specifically resected for research purposes (14). The normal pituitaries included tissue from patients with the clinical diagnosis of Cushing's disease $(n=14)$, prolactinomas $(n=3)$, acromegaly $(n=1)$, a non-functioning adenoma $(n=1)$ and an arachnoid cyst $(n=1)$. Abnormal pituitary tissue was classified as adenoma, aggressive adenoma or carcinoma. The 65 adenomas were categorised as GH-secreting tumours $(n=19)$, ACTHsecreting tumours $(n=19)$, prolactinomas $(n=7)$ and non-functioning tumours $(n=18)$. The aggressive tumours showed invasive growth and/or recurrence; this group included non-functioning adenomas $(n=5)$, prolactinomas $(n=2)$ and GH secreting tumours $(n=2)$. The pituitary carcinoma group included one ACTHsecreting tumour and two prolactinomas; these patients had histologically-verified extra-pituitary metastases.

\section{Immunohistochemistry}

Tissue preparation All tissues were collected at routine transsphenoidal surgery and prepared for pathological examination in a standard manner. Paraffin sections were cut at $3 \mu \mathrm{m}$, air-dried, then placed in a $60^{\circ} \mathrm{C}$ oven overnight. Sections were de-waxed in xylene, followed by immersion in a solution of $750 \mu \mathrm{l} 30 \%$ hydrogen peroxide and $50 \mathrm{ml}$ methanol for $10 \mathrm{~min}$ to block endogenous peroxidase. Sections were rehydrated to tap water, ready for antigen retrieval. The sections were superheated for $4 \mathrm{~min}$ in $0.01 \mathrm{M}$ citrate buffer ( $\mathrm{pH}$ 6.0), then placed in tap water immediately to avoid drying. The slides were transferred to phosphate-buffered saline (PBS), then placed in a protein-blocking solution for 10 mins before immunostaining.

Immunostaining - cyclins Immunostaining was performed using a standard avidin-biotin complex (ABC) method followed by tyramide amplification using an automated staining machine (Optimax Plus 1.5; Amenarini). Both primary antibodies anti-cyclin D1 (clone P2D11F1, Vector laboratories, Inc, Peterborough) and anti-cyclin E (clone 13A3, Vector Laboratories Inc, Peterborough) were placed on sections at 1/100 and $1 / 200$ dilutions respectively for an overnight incubation at $4{ }^{\circ} \mathrm{C}$ in a wet chamber. The following morning the sections were washed in PBS then incubated in a biotinylated anti-mouse second layer for $30 \mathrm{mins}$; the sections were again washed in PBS then incubated in the avidin-biotin complex (Vectorstain Elite Kit PK6200; Vector laboratories Inc., Peterborough, UK.). The sections were washed in PBS then incubated in a biotinylated tyramide solution (NEL 700 NEN TSA indirect Kit, Life Science Products, UK) 1/100 for $5 \mathrm{~min}$; they were then washed in PBS and the avidin-biotin complex reapplied for a further 20 mins. The tyramide step is an amplification procedure, and is used routinely in our laboratory for examination of lymphomas. After three washes in PBS the sections were visualised with activated 3'3'diaminobenzidine-tetrahydrochloride solution (DAB; Kentec Dab tablets 4170; Biostat, Stockport, UK) for 5 min.; this resulted in a brown end-product. The sections were counterstained with Gill's haematoxylin and dehydrated through graded alcohols before 
mounting in DPX. In the tumour samples, consecutive sections were immunostained for the relevant pituitary hormone to ensure the area counted for cyclin D1 and cyclin E was homogeneous for the hormone stain.

Immunostaining - pituitary hormones Pituitary hormone stains were performed to establish the hormone phenotype of the tumour samples. GH, ACTH, TSH, FSH and LH antibodies were purchased from Dako Corp. (Oxford, UK). The prolactin antibody was purchased from Novacastra (Newcastle-upon-Tyne, UK). The sections were incubated overnight in the primary antibody at $4{ }^{\circ} \mathrm{C}$. The standard $\mathrm{ABC}$ protocol was followed thereafter. A normal human pituitary was used as a positive control; the hormone antibody was omitted and replaced by mouse immunoglobulin as a negative control.

Haemotoxylin and eosin (H $\mathcal{E}$ E), and reticulin staining The sections from pituitary samples were stained with $\mathrm{H}$ and $\mathrm{E}$ as a general stain to demonstrate the various tissue components. A reticulin stain was performed to provide an indication of tissue architecture.

\section{Controls}

Positive controls Human tonsil tissue was used as a positive control since this contains lymphoid tissue with variable proliferative activity. In the basal layer of the squamous epithelium, where the cells are actively proliferating, cyclins D1 and E can readily be demonstrated as dense nuclear staining; however, in the mantle zone the cells are predominantly quiescent and no cyclin D1 or E should be detected. For cyclin D1, a mantle cell lymphoma was also used as this is known to over-express cyclin D1 (8).

Negative controls Negative controls were used in each staining batch in which the primary antibody was omitted and replaced by mouse immunoglobulin (negative control mouse immunoglobulin, Amenarini, Finchampstead, Berkshire, UK). The ABC with tyramide amplification protocol was followed thereafter. Positive and negative controls were run with every experimental section.

\section{Quantitation}

All sections were examined at x 40 magnification using bright-field microscopy. The sections were assessed by a single observer blinded as to the diagnosis. In each section, $\sim 500$ cells were analysed using a semiquantitative technique using standard measurement application software (H.O.M.E. graphics workstation, Impact Zeiss Alcatel TitN Answare-version 3.02,944221 St Bartholomew's) with Microsoft Windows. The percentage of positive and negative cells was then expressed as a ratio of positive or negative cells to the total number of cells counted, and graded as:

$$
\begin{array}{ll}
0 \% \text { positive cells }= & \text { negative } \\
<10 \% \text { positive cells }= & 1+ \\
11-25 \% \text { positive cells }= & 2+ \\
26-50 \% \text { positive cells }= & 3+ \\
>50 \% \text { positive cells }= & 4+
\end{array}
$$

\section{Statistical analysis}

The data were subjected to a non-parametric test procedure, the Kruskal-Wallis test, for overall significance of group effects, followed by the Mann-Whitney test for individual comparisons, using the Arcus Quickstat Biomedical version 1.2.

\section{Results}

Normal tonsil tissue revealed nuclear staining in the basal layer of the squamous epithelium for cyclin D1. The mantle cell lymphoma, which is known to overexpress cyclin D1, showed strong positive nuclear staining.

Overall, cyclin D1 positive nuclear expression was seen in 40 of $95(42 \%)$ cases, most of which were graded either $1+$ or $2+$. No tissue sample showed evidence of cytoplasmic staining. The normal pituitary revealed a positive count of 5/20 (25\%), all graded as $1+$. Non-functioning pituitary adenomas showed positive nuclear staining in $12 / 18(67 \%)$ of the tumours investigated. Prolactinomas demonstrated a positive count of $3 / 7(42 \%)$, graded $1+$ to $4+$ (Table $1 \mathrm{~A})$. The aggressive group showed positivity of $6 / 9(67 \%)$, graded between $1+$ and $3+$ (Table 1A). Statistically, there was a significant difference in positive counts between all pituitary groups combined $(P<0.03$; Kruskall-Wallis test). Specifically, the Mann-Whitney test revealed a significant difference between the normal pituitary group and the non-functioning adenomas $(P<0.02)$ and the aggressive group $(P<0.01)$. Combining the groups according to tumour grade (normal, adenoma, aggressive and carcinoma) showed a significant group effect $(P=0.05$; Kruskall-Wallis test), but individual comparisons (normal vs adenomas; adenomas vs aggressive) were not statistically significant.

For cyclin E, normal tonsil tissue showed intense nuclear staining in the basal layer of the squamous epithelium and positive nuclei were also noted in the proliferating cells of the germinal centres, with no expression in the quiescent cells of the mantle zone. Overall, cyclin E positive nuclear staining was seen in $13 / 95(14 \%)$ cases, most of which were graded between $1+$ and 2+. No tissue showed any evidence of cytoplasmic staining. The Kruskal-Wallis test revealed a significant difference between all pituitary groups $(P<0.02)$. The normal pituitary group revealed positive staining of $1 / 20(5 \%)$, graded $1+$. The Cushing's group revealed the highest positive count of $7 / 19(37 \%)$, 
Table 1(A) Cyclin D1 results.

\begin{tabular}{lcccccc}
\hline & $\begin{array}{c}\text { Protein } \\
0\end{array}$ & $\begin{array}{c}\text { expression } \\
1+\end{array}$ & $\begin{array}{c}\text { (no. of cases) } \\
2+\end{array}$ & $3+$ & $4+$ & P value* $^{*}$ \\
\hline Diagnosis & & & & & & \\
Normal & 15 & 5 & 0 & 0 & 0 & 0.92 \\
Acromegaly & 13 & 6 & 0 & 0 & 0 & 0.65 \\
Cushing's & 14 & 7 & 0 & 0 & 0 & 0.02 \\
NFPA & 6 & 11 & 0 & 0 & 1 & 0.23 \\
Prolactinomas & 4 & 2 & 2 & 1 & 1 & 0.01 \\
Aggressive & 3 & 1 & 0 & 0 & 0 & 1.0 \\
Carcinomas & 2 & & & 0 & 0 & \\
\hline
\end{tabular}

* Mann-Whitney test compared with normal group. The main group effect was assessed by the Kruskal-Wallis test $(P<0.03)$ The Table shows the percentage of tumours showing cyclin D1 expression according to the number of positive cells with nuclear staining. The $P$ value relates to the comparison of the tumour group as compared with the control tissue. $\mathrm{NFPA}=$ non-functioning pituitary adenomas.

Table 1(B) Cyclin E results.

\begin{tabular}{lcccccc}
\hline & $\begin{array}{c}\text { Protein } \\
0\end{array}$ & $\begin{array}{c}\text { expression } \\
1+\end{array}$ & $\begin{array}{c}\text { (no. of cases) } \\
2+\end{array}$ & $3+$ & $4+$ & P value $^{*}$ \\
\hline Diagnosis & & & & & & \\
Normal & 19 & 1 & 0 & 0 & 0 & 1.000 \\
Acromegaly & 18 & 1 & 0 & 0 & 0 & 0.03 \\
Cushing's & 12 & 2 & 0 & 0 & 0 & 0.91 \\
NFPA & 16 & 2 & 0 & 0 & 0 & 0.31 \\
Prolactinomas & 5 & 0 & 0 & 0 & 0 & 1.0 \\
Aggressive & 9 & 0 & 0 & 0 & 0 & 1.0 \\
Carcinomas & 3 & 2 & & 0 & \\
\hline
\end{tabular}

${ }^{*}$ Mann-Whitney test compared with normal group. The main group effect was assessed by the Kruskal-Wallis test $(P<0.02)$ The Table shows the percentage of tumours showing cyclin E expression according to the number of positive cells with nuclear staining. The $P$ value relates to the comparison of the tumour group as compared with the control tissue.

$\mathrm{NFPA}=$ non-functioning pituitary adenomas.

graded $1+$ to $2+$ (Table $1 \mathrm{~B})$. A significant difference between the normal pituitary group and the Cushing's group was clearly demonstrated $(P<0.03)$. These corticotroph adenomas constituted 7 microadenomas and two macroadenomas (one of these was removed in the second trimester of pregnancy).

\section{Discussion}

While our technique was clearly able to demonstrate cyclin D1 expression in control tissue, cyclin D1 was not commonly expressed by any normal or abnormal pituitary, with only a single prolactinoma showing more than $50 \%$ of cells with nuclear positivity. The majority of pituitary tumours showed either no nuclear positivity, or its presence in less than $10 \%$ of cells. It should also be noted that all pituitary tissue showed staining confined to the nucleus. This is in keeping with other data on proliferation indices in pituitary tumours, which in general show only very low rates of cell division. However, we were still able to demonstrate that pituitary tumours as a group had a larger number of samples with significant cyclin D1 positivity compared with normal pituitary, with sub-group analysis suggesting that this was principally due to the non-functioning tumours and those showing aggressive characteristics. It should be noted that many of the latter were also nonfunctioning. This may relate also to the fact that the CDK inhibitor p16 is underexpressed in some $70 \%$ of non-functioning adenomas, probably secondary to aberrant methylation of $\mathrm{CpG}$ islands within its gene (16). Pituitary carcinomas did not appear to differ from the more benign tumours. This in turn suggests that high cyclin D1 immunopositivity is an early change mainly seen in non-functioning tumours. However, it should be noted that in general non-functioning tumours are larger than other types, and it is possible that the degree of cyclin D1 expression relates to the size or degree of invasiveness of the tumour; previous work has shown an association between loss of heterozygosity at 11q13 (the approximate locus of the cyclin D1 gene) and tumour invasiveness (17). Unfortunately, the small number of tumours showing cyclin D1 positivity precluded any statistical demonstration of this possible effect.

It may be noted that our positivity rate in the normal pituitaries was $25 \%$, as compared with the complete absence of positivity seen in the normal pituitary reported by Hibberts et al. (11). However, we graded as grade 1 positive any tissue which showed $1-10 \%$ 
cells staining for cyclin D1, while in the previous study these were included in the negative group (11). Another possibility is that the discrepancy is due to the differing source of the normal pituitary in the two studies. Hibberts et al. (11) used autopsy material obtained within $6 \mathrm{~h}$ of death, while we used contemporaneous surgical material removed in the same manner as the tumour specimens. It is therefore possible that this tissue is more clearly representative of the normal pituitary, and we believe is a more appropriate control group. However, of the 5 positive 'normal' pituitaries, 4 were from patients with a clinical diagnosis of Cushing's disease and one from a patient with an arachnoid cyst. We therefore cannot entirely exclude the possibility that the positive-staining cells in such normal pituitary are actually a consequence of the primary pathology, but we feel this is unlikely. We also used a tyramide amplification step, but this did not appear to markely increase the positivity rate of the tumours compared with the previous study; overall, expression in $10 \%$ or more cells was seen in 7/75 (9\%) of tumours in our study, compared with c. $20 \%$ reported previously (11). This previous study also noted higher cyclin D1 positivity in non-functioning tumours, as also reported in a recent preliminary communication from another group (18), but found that this appeared to be unrelated to tumour invasiveness. In thyroid tumours, cyclin D1 expression was also found to be unrelated to the degree of malignancy (19).

Cyclin E positivity was seen in a minority of both normal and adenomatous tissue, with only two tumours showing more than $10 \%$ of cells as positive. There was no cytoplasmic staining in any tissue apart from occasional 'dotty' staining in some of the nonfunctioning pituitary adenomas, whose significance is unclear. There was a statistical difference in nuclear staining between the groups, but in this case it appeared to be entirely due to the corticotroph tumours, which were the only group to be distinctly different to the normal pituitary. This is particularly of interest since we have previously reported that immunostaining for the tumour suppressor gene p27 was specifically and markedly lowered in corticotroph tumours as compared with other secretory and non-secretory phenotypes (14). p27 interacts with cyclin E directly, either in a classical mode as a CDK inhibitor, or reciprocally being phosphorylated and inactivated by cyclin E (20). In either case, there appears to be an inverse correlation betwen p27 and cyclin E expression. However, rather surprisingly, the malignant pituitary tumours were also previously characterised as being p27 negative (14), but in this analysis did not clearly display cyclin E positivity. The reasons for this discrepancy are not obvious, but the sample size is small and it would be unwarranted to speculate on the basis of only three tumours.

In summary, analysis of cyclin D1 expression in a large cohort of pituitary tumours and normal pituitaries demonstrated that cyclin D1 expression was infrequent in both normal and abnormal pituitary, but more frequent staining was seen in non-functioning and aggressive adenomas. Cyclin E expression was also sparse, but in this case was over-expressed in corticotroph tumours. It is speculated that the higher rate of cyclin E positivity in corticotroph adenomas relates to the previously-described paucity of p27 in these tumours; this may in turn reflect increased degradation of p27 allowing cyclin E to increase, or conversely cyclin E increasing p27 degradation.

\section{References}

1 Sherr CJ. Mammalian G1 Cyclins. Cell 199373 1059-1065.

2 Hinds PW, Dowdy SF, Ng Eaton E, Arnold A \& Weinberg RA. Function of a human cyclin gene as an oncogene. PNAS 199491 709-713.

3 Asa SL \& Ezzat S. The cytogenesis and pathogenesis of pituitary adenomas. Endocrine Reviews 199819 798-827.

4 Pestell RG, Albanese C, reutens AT, Segall JE \& Lee RJ, Arnold A. The cyclins and cyclin-dependent kinase inhibitors in hormonal regulation of proliferation and differentiation. Endocrine Reviews $199920501-534$.

5 Jiang W, Kahn SM, Tomita N, Lu SH \& Weinstein IB. Amplification and expression of human cyclin D gene in oesophageal cancer. Cancer Research 19922 2980-2983.

6 Nishida N, Fukuda Y, Komeda T, Kita R, Sando T, Furukawa M et al. Amplification and overexpression of the cyclin D1 gene in aggressive human hepatocellular carcinoma. Cancer Research 199454 3107-3110.

7 Arnold A, Kim HG, Gaz RD, Eddy RL, Fukushima Y, Byers MG et al. Molecular cloning and chromosomal mapping of DNA rearranged with the parathyroid hormone gene in a parathyroid adenoma. Journal of Clinical Investigation 198983 2034-2040.

8 Singh N \& Wright DH. The value of immunohistochemistry on paraffin wax embedded tissue sections in the differentiation of lymphocytic from mantle cell lymphomas. Journal of Clinical Pathology 199750 16-21.

9 Dou QP, Levin AH, Zhao S \& Pardee AB. Cyclin E and cyclin A as candidates for the restriction point protein. Cancer Research 1993 53 1493-1497.

10 Keyomarsi K, O'Leary N \& Molnar G. Cyclin E: a potential prognostic marker for breast cancer. Cancer Research 199454 $380-385$.

11 Hibberts NA, Simpson DJ, Bicknell JE, Broome JC, Hoban PR, Clayton RC \& Farrell WE. Analysis of cyclin D1 (CCND1) allelic imbalance and overexpression in sporadic human pituitary tumours. Clinical Cancer Research 19995 2133-2139.

12 Lloyd R, Jin L, Qian X \& Kulig E. Aberrant p2 $7^{\text {Kip1 }}$ expression in endocrine and other tumors. American Journal of Pathology 1997 150 401-407.

13 Bamberger CM, Fehn M, Bamberger A-M, Ludecke DK, Beil FU, Saeger W \& Schulte HM. Reduced expression levels of the cell cycle inhibitor p $27^{\mathrm{Kip} 1}$ in human pituitary adenomas. European Journal of Investigation 1999140 250-255.

14 Lidhar K, Korbonits M, Jordan S, Khalimova Z, Kaltsas G, Lu X, Jenkins PJ, Monson JP, Besser GM, Lowe DG \& Grossman AB. Low expression of the cell cycle inhibitor $\mathrm{p} 27^{\mathrm{Kip} 1}$ in normal corticotroph cells, corticotroph tumors, and malignant pituitary tumors. Journal of Clinical Endocrinology and Metabolism 199984 3823-3830.

15 Dahia, PML, Aguiar RCT, Honegger J, Fahlbusch R, Jordan S, Lowe DL, Lu X. Besser GM \& Grossman AB. Mutation and expression analysis of the p27/Kip gene in corticotrophinsecreting tumours. Oncogene 199816 69-76.

16 Simpson DJ, Bicknell JE, McNicol AM, Clayton RN \& Farrell WE. Hypermethylation of the p16/CDKN2A/MTS1 gene and loss of protein expression is associated with nonfunctional pituitary 
adenomas but not somatotrophinomas. Genes Chrosomes Cancer 199924 328-336.

17 Bates AS, Farrell WE, Bicknell EJ, McNicol AM, Talbot AJ, Broome $\mathrm{JC}$ et al. Allelic deletion in pituitary adenoma reflects aggressive biological activity and potential values as a prognostic markeer Journal of Clinical Endocrinology and Metabolism 199782 818824.

18 Turner HE, Nagy Zs, Esiri MM \& Wass JAH. Expression of cyclins in pituitary adenomas. Journal of Endocrinology (Abstract Supplement) $1999163 \mathrm{P} 47$.
19 Wang S, Wuu J, Savas L, Patwardhan N \& Khan A. The role of cell cycle regulatory proteins, cyclin D1, cyclin E, and p27 in thyroid carcinogenesis. Human Pathology 199829 1304-1309.

20 Sheaff RJ, Groudine M, Gordon M, Roberts JM \& Clurman BE. Cyclin E-CDK2 is a regulator of $\mathrm{p} 27^{\mathrm{Kip} 1}$. Genes and Development $1997111464-1478$.

Received 20 March 2000

Accepted 22 May 2000 